\title{
Serum Interleukin 19 in Patients with Alopecia Areata
}

\author{
A.A.Ebrahim, A.I.Mustafa, W.A.Abd El-Haleem and A.E.Salah \\ Dermatology, Venereology and Andrology Dept., Faculty of Medicine, Benha Univ., Benha, Egypt \\ E-Mail: Sokarzyadah@gmail.com
}

\begin{abstract}
Alopecia areata (AA) is a typical ailment influencing the analgen period of the hair follicles. Interleukin (IL)- 19 is an individual from an IL-10 subfamily which additionally incorporates IL-20, IL-22 and IL-24. It normally shows as restricted, all around differentiated patches of going bald. It might present as single or different patches. The point of this examination was to survey the degree of serum of IL-19 in patients with AA to assess its part in AA pathogenesis and its conceivable connection with seriousness of the illness. Case control study included 30 patients experiencing AA notwithstanding 30 obviously sound people of coordinated age and sex as a benchmark group. Analysis of AA was made based on clinical discoveries and the seriousness of the sores will be evaluated by Severity of Alopecia Tool (SALT) score. The serum IL-19 levels estimated by chemical connected immunosorbent examine (ELISA). We found that all out AA bunch indicated fundamentally higher serum IL-19 when contrasted with control gathering. Higher IL-19 was essentially connected with dynamic cases. Likewise, it expanded steadily with seriousness. Something else, no noteworthy affiliations were found with respect to IL-19 level as indicated by sexual orientation, family ancestry, beginning, course, assortment, repeat and past treatment in AA gathering.
\end{abstract}

Keywords: Alopecia areata, Interleukin-19.

\section{Introduction}

Alopecia areata is a generally basic reversible balding issue. It is typically showed as sketchy zones of complete balding on the scalp and other body parts that can advance to finish loss of all body hair. The etiology of AA is obscure yet it is portrayed by hair cycle brokenness and the presence of peribulbar and perifollicular mononuclear cell invades [1].

It is a kindhearted condition and most patients are asymptomatic; notwithstanding, it can cause extensive passionate and psychosocial worry in influenced people especially in serious or ongoing cases. It regularly shows as restricted, all around outlined patches of going bald [2].

Notwithstanding safe capacity aggravation, hereditary and natural components assume a function in its pathogenesis. The perplexing connections between inclining hereditary and natural factors probably add to the acceptance of insusceptible interceded reactions in AA [3].

Alopecia areata is a typical malady that influences roughly $0.1-0.2 \%$ of all populace. The problem influences youngsters, people of all hair tones. The most noteworthy pervasiveness is seen between the second and fourth many years of life. Up to $66 \%$ of patients are under 30 years of age, while just $20 \%$ are above 40years old [4]

Interleukin-19 is an individual from an IL-10 subfamily which likewise incorporates IL-20, IL-22 and IL-24 [5]. It has novel properties including articulation just as target cells outside of the resistant framework and $\mathrm{NF}-\kappa \mathrm{B}$-free impacts. Likewise, it is communicated by epithelial cells with supportive of provocative incitement [6].

Interleukin-19 neglected to initiate the creation of any immunoglobulin [7]. Hair follicle explicit immunoglobulin neutralizer focus is expanded in the fringe blood of AA patients [8].
The point of this investigation was to survey the degree of serum of IL-19 in patients with AA to assess its function in AA pathogenesis and its conceivable relationship with seriousness of the malady.

\section{Materials and methods}

The investigation was led as a case control study. The investigation remembered 30 for 30 patients experiencing AA. Furthermore, 30 clearly sound people of coordinated age and sex were picked as a benchmark group. All Patients were chosen from the outpatient facility of Dermatology and Andrology Department of Benha University Hospitals on the period from December 2019 to February 2020 .

Consideration measures were patients with AA and the conclusion will be founded on clinical discoveries and the seriousness of the injuries will be surveyed by SALT score .

Rejection measures were Patients with AA experiencing limited scalp contaminations/irritation, incendiary skin illness, immune system sickness, fundamental ailment, dangerous tumors and pregnant and lactating female patients.

Diagnosis of AA was made on the basis of clinical findings and the severity of the lesions will be assessed according to SALT score.

Severity of AA lesions was assessed using SALT score. SALT score is a mathematical approach to determine percentage of hair loss in the scalp. The scalp is divided into the following 4 areas:- Vertex: $40 \%(0.4)$ of scalp surface area, Right profile of scalp: $18 \%(0.18)$ of scalp surface area, Left profile of scalp: $18 \%(0.18)$ of scalp surface area and Posterior aspect of scalp: $24 \%$ (0.24) of scalp surface area.

Five milliliters of venous blood sample were collected from every participant in the study under complete aseptic precautions in the plain test tubes without anticoagulant. After coagulation, samples were centrifuged (at $1500 \mathrm{~g}$ for $15 \mathrm{~min}$ ). The separated serum 
was aliquoted and stored at $-20^{\circ} \mathrm{C}$ for subsequent assay of IL-19.

Measurement of serum IL-19 was done by human IL19 immunoassay using a double-antibody sandwich ELISA.

\section{Results}

Alopecia areata group showed significantly higher IL-19 level when compared to control group (mean=204.1 versus 14.8, $\mathrm{p}<0.001$ ).

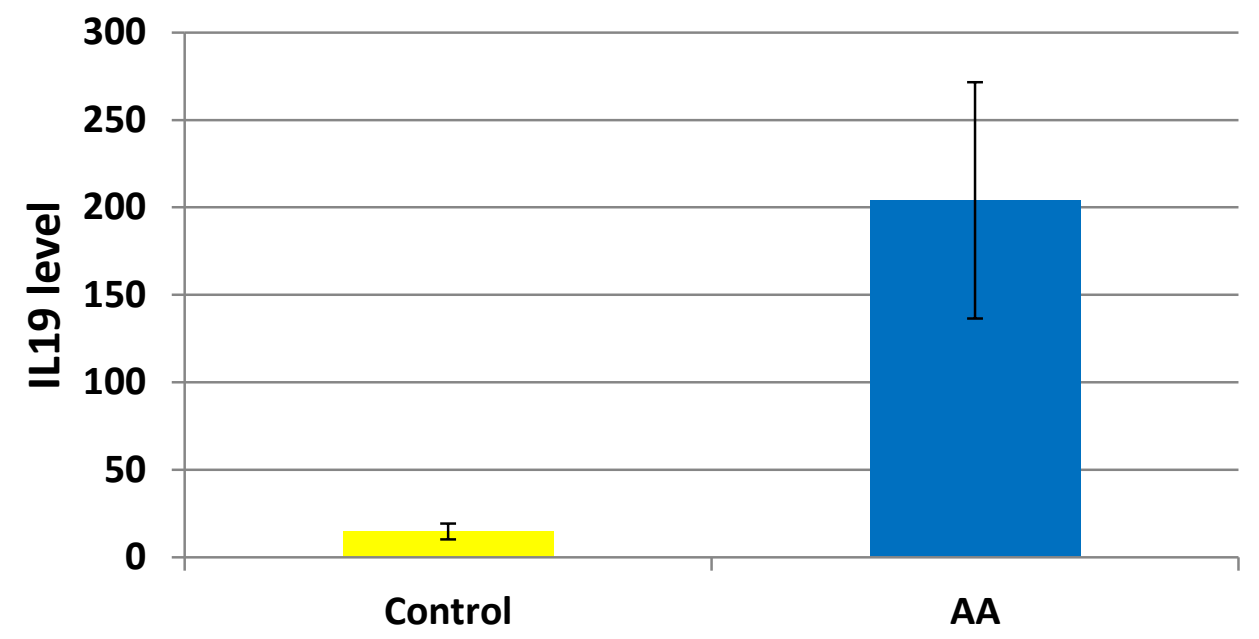

Fig (1) Bar chart for IL-19 level in all studied groups. Bar represents mean, while error bar represents standard deviation.

Table (1) Comparison of IL-19 level among all studied groups.

\begin{tabular}{|c|c|c|c|c|c|}
\hline & \multicolumn{2}{|c|}{$\begin{array}{c}\text { Control } \\
\mathbf{N}=\mathbf{2 0}\end{array}$} & \multicolumn{2}{|c|}{$\begin{array}{c}\mathrm{AA} \\
\mathrm{N}=\mathbf{4 0}\end{array}$} & \multirow[t]{2}{*}{ p } \\
\hline & mean & \pm SD & mean & \pm SD & \\
\hline IL-19 & 14.8 & \pm 4.5 & 204.1 & \pm 67.5 & $<0.001$ \\
\hline
\end{tabular}

SD, standard deviation.

\section{Discussion}

Alopecia areata is a typical infection influencing the analgen period of the hair follicles. It is considered as an immune system issue [9]. It is viewed as a T-cellinterceded autoimmunity happening in hereditarily inclined people [10]. It usually shows as confined, very much delineated patches of balding. It might present as single or numerous patches. Little unmistakable patches may union and structure bigger patches [11].

The intricate connections between inclining hereditary and ecological factors probably add to the acceptance of resistant intervened reactions in AA [3].

The examination was directed as a case control study. The examination remembered 30 for 30 patients experiencing AA. Furthermore, 30 obviously solid people of coordinated age and sex were picked as a benchmark group.

All patients were exposed to finish history, complete general assessment including, dermatological clinical assessment and assessment of serum level IL-19 in patients and control bunches by ELISA.

In the current investigation, AA bunch indicated altogether higher IL-19 level when contrasted with control gathering.
Interleukin-19 assumes an essential function in down-guideline of $\mathrm{T}$ cell-interceded insusceptible reactions [12]. It energizes versatile invulnerability by advancing $\mathrm{Th} 2$ reactions in a positive input circle expanding IL-4-positive and less IFN $\gamma$-positive leukocytes [13]. It assumes some significant parts in fiery reactions since it can actuate $\mathrm{TNF}-\alpha$ creation in monocytes [14]. In sera and lesional biopsy tests from patients with AA, Th1 cytokines, IFN- $\gamma$ and TNF- $\alpha$ have been discovered [15].

Interleukin-19 is delivered by keratinocytes, it upregulates the creation of keratinocyte development factor from CD8+ T cells [16]. Furthermore, because of the cytotoxic idea of most CD8+ T cells, their quality inside hair follicles could undoubtedly upset the development of hair [17]. The intermingling of articulation information from the AA model with the human investigations affirm that antigen introduction in relationship with cytotoxic $\mathrm{T}$ lymphocyte antigen-4 (CTLA-4)- CD80/86 co-incitement is embroiled in the advancement of AA [18]. 


\section{References}

[1] L.Strazzulla, E.Wang, L.Avila, Alopecia areata: an appraisal of new treatment approaches and overview of current therapies. JAAD,Vol.78(1), PP.15-24, 2018.

[2] M.Gonul, B.Cemil, H.Ayvaz, Comparison of quality of life in patients with androgenetic alopecia and alopecia areata. Anais brasileiros de dermatologia, Vol.93(5), PP.651-658, 2018.

[3] H.Almohanna, A.Ahmed and J.Tsatalis ,The Role of Vitamins and Minerals in Hair Loss: A Review. Dermatol Therapy; Vol.9, PP.51-70,2019.

[4] E.Perera, Y.Yip and R.Sinclair, Alopecia Areata. Hair Ther Transplant, Vol. 4, PP.1-5,2014.

[5] H.Oral, S.Kotenko, M.Yilmaz, Regulation of T cells and cytokines by the interleukin-10 (IL-10)-family cytokines IL-19, IL-20, IL-22, IL-24 and IL-26. Eur J Immunol, Vol.36, PP.380-388,2006.

[6] C.Fielding, Interleukin-19: A new target to aim for? Rheumatol, Vol.51(3), PP.399-400, 2012.

[7] L.Hummelshoj, L.Ryder and L.Poulsen, The Role of the interleukin-10 Subfamily Members in Immunoglobulin Production by Human B Cells. J Immunol, Vol.64, PP.40-47, 2006.

[8] Fuchs , Enteroviruses as the cause of a variety of chronic diseases. Allgemeine Homöopathische Zeitung, Vol.262(02), PP.04-01, 2017.

[9] S.Gheida and G.Soliman, Evaluation of macrophage migration inhibitory factor in patients with alopecia areata before and after the treatment. Egy J Dermatol Venereol, Vol.38(2), PP.52-58, 2018.
[10] G.Ozaydin-Yavuz, I.Yavuz, Alopecia areata different view; Heavy metals. Indian J Dermatol, Vol.64, PP. 7-11, 2019.

[11] K.Seetharam ,Alopecia areata: An update. IJDVL, Vol.79 (5), PP. 563-575, 2013.

[12] R.Maizels, A.Balic, N.Gomez-Escobar, Helminth parasites-masters of regulation. Immunol Rev, Vol.201, PP.89-116, 2004.

[13] G.Gallagher, Interleukin-19: multiple roles in immune regulation and disease. Cytokine Growth Factor Rev, Vol.21, PP.345-352, 2010.

[14] S.Liao, Y.Cheng, Y.Wang, IL-19 induced Th2 cytokines and was up-regulated in asthma patients. J Immunol, Vol.173, PP.6712-6718, 2004.

[15] Y.Gohary and D.Fattah , Detection of tumor necrosis factor-alpha in nonlesional tissues of alopecia areata patients: A prove for a systemic disease. Int J Trichol, Vol.9(4), PP.150-154, 2017.

[16] E.Witte, G.Kokolakis, K.Witte, IL-19 is a component of the pathogenetic IL-23/IL-17 cascade in psoriasis. J. Invest. Dermatol, Vol.134, PP.27572767,2014.

[17] G.Celik and M.Gonul,Alopecia Areata: What is New in Epidemiology, Etiopathogenesis Clinical Aspects and Prognosis. Chapter 5, Hair Disorder,Vol.22, PP.1-33,2018.

[18] J. Sundberg, K.McElwee, J.Carroll, Hypothesis Testing: CTLA4 Co-stimulatory Pathways Critical in the Pathogenesis of Human and Mouse Alopecia Areata. J Invest Dermatol; Vol.131(11), PP. 23232324,2011. 